\title{
The prognostic value of left atrial and left ventricular strain in patients after ST-segment elevation myocardial infarction treated with primary percutaneous coronary intervention
}

\author{
Ai-Ai Chu ${ }^{1,2}$, Ting-Ting $\mathrm{Wu}^{1}$, Lu Zhang ${ }^{1}$, Zheng Zhang ${ }^{1}$ \\ ${ }^{1}$ Heart Center, The First Hospital of Lanzhou University, Lanzhou University, Lanzhou, China \\ ${ }^{2}$ Department of Cardiology, Gansu Provincial Hospital, Lanzhou, China
}

\begin{abstract}
Background: Global longitudinal strain (GLS) based on two-dimensional speckle-tracking echocardiography (2D-STE) might better reflect left ventricular $(L V)$ contractile performance than conventional parameters. Recently, left atrial (LA) strain has been used as a more accurate alternative to assessing $L A$ performance. The aim in this study was to assess the clinical prognostic value of left ventricular GLS (LV GLS) and peak atrial longitudinal strain (PALS) in patients after ST-segment elevation myocardial infarction (STEMI).

Methods: The study enrolled 199 patients who underwent primary percutaneous coronary intervention ( $p P C I$ ) for first STEMI. Conventional and $2 D$-STE were performed within $48 \mathrm{~h}$ after pPCI. LV GLS and PALS were related to $L V$ remodeling at 6-month follow-up and to adverse events.

Results: Diabetes mellitus, GLS and PALS independently predicted LV remodeling. With multivariable Cox proportional hazards, diabetes mellitus, GLS and PALS were predictive of adverse clinical outcomes. However, PALS did not add significant incremental value beyond LV GLS in the prediction of LV remodeling (increase in area under the receiver-operator characteristic curve [AUC]: 0.05, $p=0.24$ ) and clinical events (even a decrease in AUC: $0.03, p=0.69$ ).

Conclusions: Both GLS and PALS provide independent prognostic value for adverse $L V$ remodeling and clinical outcomes after STEMI. However, the ability of the combination of PALS and GLS to predict $L V$ remodeling and clinical outcomes may not be superior to that of a single indicator. (Cardiol J 2021; 28, 5: 678-689)
\end{abstract}

Key words: acute myocardial infarction, atrial strain, global longitudinal strain, echocardiography, remodeling, prognosis

\section{Introduction}

It is well known that outcomes of ST-segment elevation myocardial infarction (STEMI) have dramatically improved in recent years because of the introduction of modern thrombolytic drugs and percutaneous coronary intervention (PCI). However, left ventricular (LV) remodeling still occurs in $30-35 \%$ of patients $[1,2]$. There is a progressive change in myocardial wall and ventricular structure, including expansion in the infarct region, wall thinning, and ventricular dilation in the non-infarcted region [3], which may be followed by adverse cardiovascular events and an increase mortality rate [4]. The introduction of two-dimensional speckle-tracking echocardiography (2D-STE) may

Address for correspondence: Zheng Zhang, PhD, Heart Center, The First Hospital of Lanzhou University, Lanzhou University, No. 1 Donggang West Road, Chengguan District, Lanzhou 730000, China, tel: +86-13919405976, fax: +86-13919405976, e-mail: zhangzhegnccu123@126.com

Received: 1.08.2019 Accepted: 20.01.2020 Early publication date: 5.02.2020

This article is available in open access under Creative Common Attribution-Non-Commercial-No Derivatives 4.0 International (CC BY-NC-ND 4.0) license, allowing to download articles and share them with others as long as they credit the authors and the publisher, but without permission to change them in any way or use them commercially. 
contribute to quantification of LV global and regional systolic function [5]. Previous studies have shown that global longitudinal strain (GLS) can be used to predict $\mathrm{LV}$ remodeling and cardiovascular events after STEMI [6-9]. However, some studies showed that like GLS, global circumferential strain (GCS) and circumferential strain rate are independent predictors of LV remodeling [10].

Left atrial (LA) volumes and LA function have been recognized as significant predictors of adverse events in a range of cardiovascular diseases $[11,12]$. Recently, 2D-STE is shown to be feasible for measuring LA deformations, thus allowing analysis of LA reservoir function (peak atrial longitudinal strain [PALS]) during the LV systolic phase [13]. More recently, LA reservoir function measured by PALS has shown good predictive value, even independently of LV GLS and LA volume [14, 15]. However, the additional value of PALS in patients with decreased LV GLS is questionable. A previous study proved that the prognostic value of PALS in patients with acute myocardial infarction (AMI) is dependent on LV GLS and LA size [16].

Accordingly, the purpose of this study was to examine patients with STEMI in: the clinical and prognostic importance of both LV GLS and PALS on LV remodeling and clinical outcome and prognostic information incremental of PALS to clinical data as well as reduced LV GLS.

\section{Methods}

\section{Study population}

In this prospective study, a total of 216 patients diagnosed with STEMI treated with primary PCI (pPCI) were enrolled from September 2017 to March 2018. The inclusion criteria were as follows: age 18 to 80 years, STEMI with onset of pain $<12 \mathrm{~h}$ before pPCI, and admission with STEMI based on present guidelines [17]. The exclusion criteria were: previous myocardial infarction or coronary artery bypass, significant valvular dysfunction, ventricular arrhythmia, atrial fibrillation or paced rhythm, and noncardiac disease with a life expectancy of $<1$ year.

All patients were treated according to present cardiology guidelines. Before pPCI, they were given a loading dose of acetylsalicylic acid (ASA), $600 \mathrm{mg}$ of clopidogrel, and $100 \mathrm{IU} / \mathrm{kg}$ of heparin (maximum 5,000 IU). This prospective study was approved by the Ethics Committee of the First Hospital of Lanzhou University. All patients signed informed consent forms.

\section{Echocardiography}

Echocardiographic data were obtained using the EPIQ 7C (Kininklijke Philips NV, Eindhoven, The Netherlands). Echocardiographic images were obtained by recording three consecutive heart cycles during apnea according to the guidelines of the American Society of Echocardiography [5]. Two experienced observers performed all patient views offline using an echocardiographic analysis system (QLAB Advanced Tissue Motion Quantification, Phillips).

Left ventricular end-systolic volume (LVESV), LV end-diastolic volume (LVEDV) and LV ejection fraction (LVEF) were determined using the biplane Simpson method in 4-, 3-, and 2-chamber views. The $L V$ was divided into 16 segments, and segments were graded $(1=$ normokinetic, 2 = hypokinetic, 3 = akinetic, $4=$ dyskinetic) according to subjective assessments of wall motion amplitude and changes in LV thickness at systole. The wall motion score index (WMSI) was defined as the sum of the segment score ratings divided by the number of segments scored. Pulsed-wave Doppler variables were measured by placing at the tip of the mitral valve (MV) leaflets from the apical 4-chamber view during diastole. The peak velocity of early (E) and late $(\mathrm{A})$ diastole and the MV deceleration time were measured, and the $\mathrm{E} / \mathrm{A}$ ratio was calculated. The measurements of myocardial peak early velocity (e') were performed at the lateral and medial mitral annulus. E/e' were obtained by dividing $\mathrm{E}$ by e'.

\section{LV strain analysis}

Two-dimensional echocardiographic images were obtained from 4-, 3-, and 2-chamber and midventricular short-axis views with frame rates of 60 to $90 \mathrm{frame} / \mathrm{s}$. 2D-STE was performed using the commercially available software QLAB Advanced Tissue Motion Quantification (Philips) equipped with STE analysis. The LV endocardial and epicardial borders were initially traced at enddiastole, and the software automatically tracked the region of interest of the myocardium. Longitudinal peak systolic strain (LPSS), was obtained for each segment from which the software provided strain curves in all 16 segments. The GLS was calculated as the average of the observed segmental values of LPSS from the apical 4-, 3-, and 2-chamber view (Fig. 1A). For LV circumferential peak systolic strain and radial peak systolic, 2D-STE analyses were performed on the LV short-axis midventricular view. Global circumferential strain and global radial strain were calculated as the mean of values from LV short-axis views. 


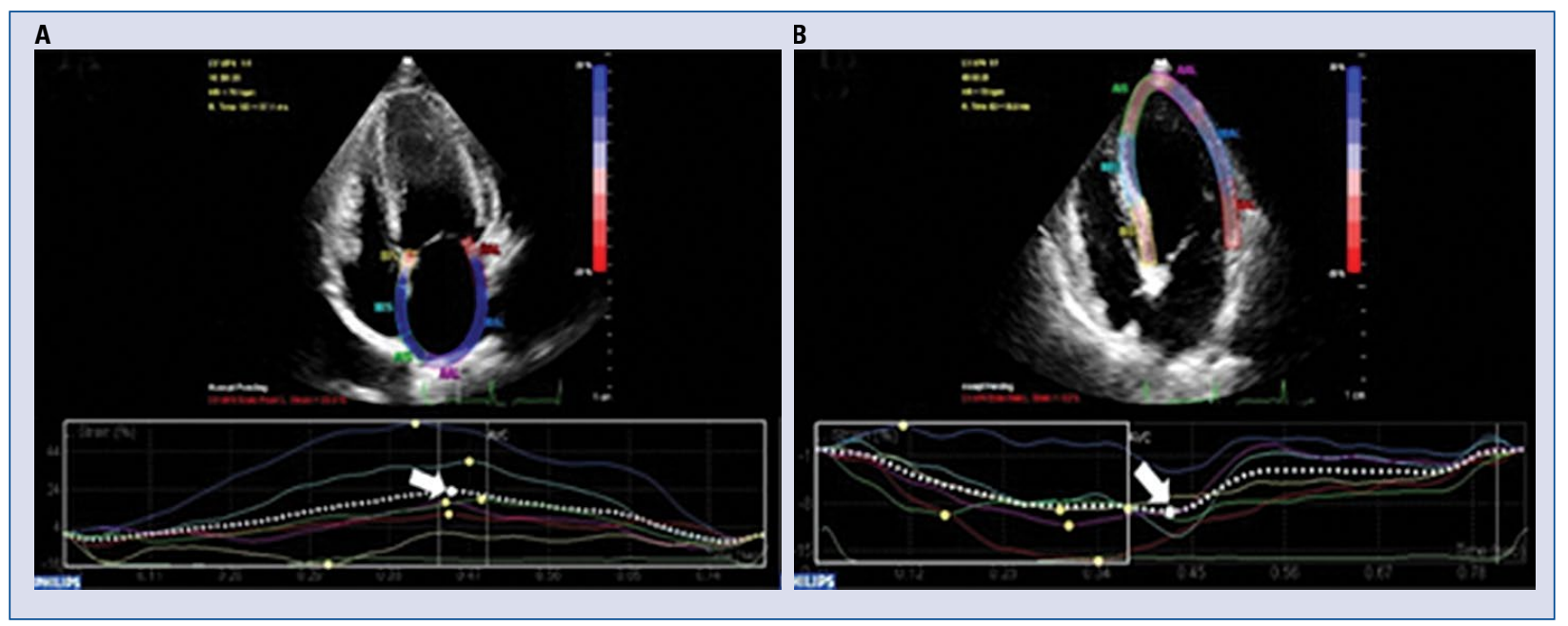

Figure 1. Two-dimensional speckle-tracking of the left ventricle (LV). The resulting strain curves for LV are shown with markings corresponding to peak global longitudinal strain (A); the resulting strain curves for left atrium are shown with markings corresponding to peak atrial longitudinal strain (B).

\section{LA function analysis}

The biplane Simpson method was used to analyze LA function. LA volume at LV end-systole (LAVmax), LA volume at LV end-diastole (LAVmin), and LA volume before atrial active contraction at the onset of the P-wave (LAVpreA) were obtained from apical 4- and 2-chamber views. All LA volumes were indexed to the body surface area [5]. From these volumes, the indexes of LA mechanical function were calculated: (1) total atrial emptying fraction: LA total ejection fraction $=(($ LAVmax - LAVmin $) /$ LAVmax $) \times 100$; (2) active atrial emptying fraction-an index of LA active contraction: LA active ejection fraction $=$ $=((\mathrm{LAVpreA}-\mathrm{LAVmin}) / \mathrm{LAVpreA}) \times 100 ;(3)$ passive atrial emptying fraction-an index of LA conduit function: LA passive ejection fraction $=((\mathrm{LAVmax}$ - LAVpreA) / LAVmax) $\times 100$; (4) atrial expansion index of reservoir function: LA expansion index $=$ $=(\mathrm{LAV} m a x-\mathrm{LAV} \min ) / \mathrm{LAV} \min \times 100$ [18]

For 2D-STE analysis of LA function, 2D grayscale images were obtained in apical 4- and 2-chamber views, consistent with software and version for analyzing LV strain. To measure PALS (LA reservoir function), the beginning of QRS wave of the electrocardiogram was used as a reference point [13]. After selecting the cardiac cycle, the LA endocardial border was manually traced, automatically creating a region of interest to cover the thickness of LA myocardium from a total of 12 atrial segments (Fig. 1B). PALS values were estimated in each LA segment from two apical views, and the mean of global PALS was calculated. Patients in whom more than two segments with poor images could not be analyzed were excluded [2].

\section{Follow-up and endpoint definition}

At least 6 months after STEMI $(18.3 \pm 5.0$ months), conventional echocardiography was performed. LV remodeling assessed by echocardiography was defined as an LVEDV increase of $>20 \%$ compared with baseline echocardiographic data [2]. Cardiovascular medical professionals completed follow-up phone calls in all patients each month after discharge from the hospital. Major adverse clinical events were a composite of death from any cause, hospitalization for heart failure and reinfarction, which were determined by both clinical visits and telephone calls. Hospitalization for heart failure occurring because of exacerbation of exertional dyspnea, with typical symptoms of pulmonary congestion and initiation of intravenous diuretics. Reinfarction was defined as a typical sign of chest pain, elevated cardiac enzyme levels, and obvious changes on the electrocardiogram [19].

\section{Statistical analysis}

Data for continuous variables are presented as the mean \pm standard deviation or median and interquartile range, and categorical variables are presented as frequencies and percentages. Continuous variables are compared using the 
independent-samples t test. Categorical variables were compared by the $\chi^{2}$ test. To examine determinants of $\mathrm{LV}$ remodeling as a dependent variable, logistic forward regression analysis was applied. Univariate analysis was performed to choose the independent variables, and those variables with borderline values $(\mathrm{p}<0.10)$ were submitted for multivariate analysis. The ability of clinical and echocardiographic parameters to predict adverse events were tested in univariate Cox proportional hazards models. To estimate the independent prognostic value of the above parameters, multivariate Cox proportional hazards analysis was also performed. Receiver operating characteristic (ROC) curve analysis were constructed, and areas under curves (AUC) were measured to determine cutoff values with maximum sensitivity and specificity. All statistical tests were two-sided, and a $\mathrm{p}$ value $<0.05$ was considered statistically significant.

\section{Results}

\section{Subject characteristics}

A total of 216 patients with their first acute STEMI treated with pPCI were initially evaluated. Seventeen patients were excluded: before echocardiographic examination, $2(0.9 \%)$ patients died during hospitalization, and $5(2.3 \%)$ patients were not available to undergo echocardiography due to poor cooperation. Another 10 (4.6\%) patients did not have sufficient image quality for tracking of the LV and LA walls. No patients were lost to follow-up. Thus, 199 patients were enrolled in the present study. Mean age was $57.4 \pm 10.7$ years, and 150 were males.

\section{Prediction of $\mathrm{LV}$ remodeling at 6 months}

At 6-month follow-up, the incidence of adverse LV remodeling was $25 \%$. The baseline characteristics and echocardiographic parameters of both the LV remodeling group and the non-LV remodeling group are summarized in Table 1. Except for diabetes mellitus, the incidence of risk factors associated with cardiovascular disease did not differ significantly between the two groups. Anterior wall STEMI appeared in $106(52 \%)$ patients and was the most common (76\%) kind of adverse LV remodeling. After immediate pPCI therapy, a comparison of echocardiographic data showed larger LVEDV, LVESV and LA volume index (LAVI); lower LVEF, LA total ejection fraction, LA active emptying fraction and LA reservoir function and higher WMSI were observed in the LV remodeling group. There were significant reductions in both LV GLS and GCS, as well as in PALS, regardless of myocardial infarction location.

Univariate analysis demonstrated the variables to be correlated to the LV remodeling, namely diabetes mellitus, creatinine kinase-MB, LAVI, LA total ejection fraction, LA active emptying fraction, LA reservoir function, PALS, WMSI, GLS and GCS. Therefore, these parameters were included in a forward stepwise multivariate analysis, and diabetes mellitus, GLS and PALS were demonstrated to independently predict $\mathrm{LV}$ remodeling (Table 2 ).

The AUC for LV GLS and PALS were 0.86 and 0.89 , respectively. However, PALS did not add significant incremental value beyond LV GLS (AUC increased from 0.86 to $0.91 ; \mathrm{p}=0.24$ ) in the prediction of $\mathrm{LV}$ adverse remodeling. The best cutoff values of LV GLS and PALS for LV remodeling were $-11.3 \%$ (sensitivity: $71.4 \%$, specificity: $84.0 \%$ ) and $28.9 \%$ (sensitivity: $72.7 \%$, specificity: $87.8 \%$ ) (Fig 2A-C).

\section{Clinical events during follow-up}

During a mean follow-up of $18.3 \pm 5.0$ months, $23(11.6 \%)$ patients reached one or more composite endpoints: 3 (1.5\%) patients died, $9(4.5 \%)$ patients had reinfarction, and $11(5.5 \%)$ patients required hospital admission to control heart failure symptoms, who were in the event group; the other 176 patients were divided into the event-free group. Comparison of clinical and echocardiographic features between patients who achieved the composite endpoint and those who did not are displayed in Table 3 .

Diabetes mellitus, LAVI, LA total ejection fraction, LA active emptying fraction, LA reservoir function, PALS, LVEF, LV GLS and GCS were univariable predictors of adverse events. All these parameters were included in a multivariate Cox proportional hazards model, and diabetes mellitus, LV GLS and PALS were independently associated with the composite events (Table 4)

The AUC for LV GLS and PALS were 0.86 and 0.83 , respectively. Similarly, PALS did not add significant incremental value beyond LV GLS (AUC decreased from 0.86 to $0.83 ; \mathrm{p}=0.69$ ) in the prediction of the composite event. The best cutoff values of LV GLS and PALS for LV remodeling were $-12.3 \%$ (sensitivity: $95.7 \%$, specificity: $67.0 \%$ ) and $28.9 \%$ (sensitivity: $88.1 \%$, specificity: $65.2 \%$ ) (Fig. 3A-C).

Figure $4 \mathrm{~A}, \mathrm{~B}$ showed survival curves by the Kaplan-Meier analysis for patients divided by the best value of LV GLS and PALS: patients with LV 
Table 1. Baseline characteristics of patients with and without left ventricular remodeling.

\begin{tabular}{|c|c|c|c|}
\hline Parameter & Non-remodeling $(n=150)$ & Remodeling ( $n=49$ ) & $\mathbf{P}$ \\
\hline \multicolumn{4}{|l|}{ Clinical parameters } \\
\hline Number & $150(75 \%)$ & $49(32 \%)$ & \\
\hline Male & $73 \%$ & $75.5 \%$ & 0.76 \\
\hline Age [years] & $57.9 \pm 10.5$ & $55.9 \pm 11.0$ & 0.26 \\
\hline $\mathrm{BMI}\left[\mathrm{kg} / \mathrm{m}^{2}\right]$ & $24.5 \pm 3.7$ & $24.7 \pm 3.9$ & 0.75 \\
\hline Diabetes & $18(12.0 \%)$ & $19(38.8 \%)$ & 0.001 \\
\hline Hypertension & $65(43.3 \%)$ & $18(36.7 \%)$ & 0.42 \\
\hline Hyperlipidemia & $53(35.3 \%)$ & $18(36.7 \%)$ & 0.86 \\
\hline Smoking & $102(68 \%)$ & $32(65.3 \%)$ & 0.73 \\
\hline Systolic BP [mmHg] & $111.2 \pm 16.9$ & $110.5 \pm 17.3$ & 0.82 \\
\hline Diastolic BP [mmHg] & $73.1 \pm 14.8$ & $69.1 \pm 9.6$ & 0.07 \\
\hline Heart rate $[\mathrm{bpm}]$ & $74.4 \pm 15.3$ & $74.5 \pm 7.4$ & 0.95 \\
\hline QRS width [ms] & $97.9 \pm 16.4$ & $102.2 \pm 21.1$ & 0.14 \\
\hline S-TO-B [min] & $328.0 \pm 174.4$ & $383.9 \pm 175.6$ & 0.053 \\
\hline D-TO-B [min] & $49.1 \pm 19.1$ & $53.2 \pm 21.2$ & 0.20 \\
\hline eGFR $\left[\mathrm{mL} / \mathrm{min} / 1.73 \mathrm{~m}^{2}\right]$ & $92.1 \pm 27.3$ & $99.0 \pm 28.8$ & 0.13 \\
\hline Creatinine $[\mu \mathrm{mol} / \mathrm{L}]$ & $71.9 \pm 26.3$ & $67.6 \pm 11.5$ & 0.19 \\
\hline Grace (scores) & $95.8 \pm 26.7$ & $98.7 \pm 22.6$ & 0.49 \\
\hline Crusade (scores) & $22.7 \pm 13.2$ & $19.9 \pm 11.8$ & 0.26 \\
\hline CK-MB [ng/mL] & $332.2 \pm 143.4$ & $436.2 \pm 117.9$ & 0.001 \\
\hline CK-MB peak time after onset [h] & $15.5 \pm 5.1$ & $19.4 \pm 5.2$ & 0.001 \\
\hline Killip class $\geq$ II & $14(9.3 \%)$ & $6(12 \%)$ & 0.56 \\
\hline Anterior wall MI & $66(44.0 \%)$ & $37(75.5 \%)$ & 0.001 \\
\hline ST max before PCl [mm] & $3.8 \pm 2.0$ & $4.5 \pm 2.4$ & 0.07 \\
\hline Multivessel coronary disease & $42(28 \%)$ & $20(41 \%)$ & 0.09 \\
\hline \multicolumn{4}{|l|}{ Medication during hospitalization } \\
\hline ASA & $150(100 \%)$ & $49(100 \%)$ & 1 \\
\hline Clopidogrel/Ticagrelor & $150(100 \%)$ & $49(100 \%)$ & 1 \\
\hline Beta-blockers & $113(75 \%)$ & $35(71 \%)$ & 0.59 \\
\hline ACEI/ARB & $89(59 \%)$ & $29(59 \%)$ & 0.99 \\
\hline Statins & $135(90 \%)$ & $45(92 \%)$ & 0.70 \\
\hline \multicolumn{4}{|l|}{ Initial LV function } \\
\hline LVESV [mL] & $86.9 \pm 21.6$ & $104.4 \pm 28.7$ & 0.001 \\
\hline LVEDV [mL] & $41.3 \pm 13.2$ & $56.6 \pm 17.6$ & 0.001 \\
\hline LVEF [\%] & $52.9 \pm 4.5$ & $46.3 \pm 3.8$ & 0.001 \\
\hline WMSI & $1.31 \pm 0.1$ & $1.37 \pm 0.1$ & 0.001 \\
\hline Deceleration time [ms] & $171.3 \pm 39.2$ & $159.0 \pm 53.4$ & 0.09 \\
\hline E/A ratio & $0.9 \pm 0.3$ & $0.9 \pm 0.5$ & 0.47 \\
\hline$E / E^{\prime}$ & $11.8 \pm 3.1$ & $12.5 \pm 3.8$ & 0.21 \\
\hline Moderate or severe MR & $6(4 \%)$ & $4(8 \%)$ & 0.06 \\
\hline GLS [\%] & $-14.7 \pm 2.9$ & $-10.6 \pm 2.4$ & 0.001 \\
\hline GCS [\%] & $-14.5 \pm 3.5$ & $-12.7 \pm 2.9$ & 0.001 \\
\hline GRS [\%] & $39.1 \pm 8.6$ & $38.7 \pm 7.8$ & 0.75 \\
\hline \multicolumn{4}{|l|}{ LA function } \\
\hline LAVI $\left[\mathrm{mL} / \mathrm{m}^{2}\right]$ & $26.8 \pm 5.0$ & $32.8 \pm 7.5$ & 0.001 \\
\hline LA total ejection fraction [\%] & $54.9 \pm 6.0$ & $52.4 \pm 5.4$ & 0.01 \\
\hline
\end{tabular}


Table 1 (cont.). Baseline characteristics of patients with and without left ventricular remodeling.

\begin{tabular}{lccc}
\hline Parameter & Non-remodeling $(\mathbf{n}=\mathbf{1 5 0})$ & Remodeling $(\mathbf{n}=\mathbf{4 9})$ & $\mathbf{P}$ \\
\hline LA passive emptying fraction [\%] & $28.3 \pm 8.1$ & $28.2 \pm 5.6$ & 0.95 \\
LA active emptying fraction [\%] & $36.9 \pm 6.6$ & $33.7 \pm 4.9$ & 0.002 \\
LA reservoir function [\%] & $125.7 \pm 31.2$ & $112.8 \pm 25.7$ & 0.01 \\
PALS [\%] & $32.5 \pm 5.9$ & $23.0 \pm 4.8$ & 0.001 \\
Follow-up LV function & & & 0.001 \\
LVESV [mL] & $88.8 \pm 23.1$ & $131.2 \pm 35.1$ & 0.001 \\
LVEDV [mL] & $39.1 \pm 15.3$ & $74.2 \pm 23.4$ & 0.001 \\
LVEF [\%] & $56.5 \pm 5.8$ & $43.9 \pm 3.9$ & \\
Composite endpoint during follow-up & & $14(29 \%)$ & 0.001 \\
Total number of complications & $9(6.0 \%)$ & & \\
\hline
\end{tabular}

Data are expressed as mean \pm standard deviation or number (\%). ACEI — angiotensin-converting enzyme inhibitors; ARB - angiotensin II receptor blocker; ASA - acetylsalicylic acid; BMI — body mass index; BP — blood pressure; CK - creatine kinase; D-TO-B - door-to-balloon time; E/A - mitral inflow peak early velocity/mitral inflow peak late velocity; $E / E^{\prime}$ - mitral inflow peak early velocity/mitral annular peak early velocity; eGFR - estimated glomerular filtration rate; GCS — global circumferential strain; GLS - global longitudinal strain; GRS - global radial strain; LA — left atrium; LAVI - left atrium volume index; LV — left ventricular; LVEDV — left ventricular end-diastolic volume; LVEF — left ventricular ejection fraction; LVESV — left ventricular end-systolic volume; MI — myocardial infarction; MR — mitral regurgitation; PALS - peak atrial longitudinal strain; ST max - maximum ST-segment elevation from a single lead; S-TO-B - symptom-to-balloon time; WMSI - wall motion score index

Table 2. Factors predicting adverse left ventricular remodeling after 6-month follow-up in univariate and multivariate analysis.

\begin{tabular}{lccc}
\hline Parameters & OR & $95 \% \mathbf{C l}$ & P \\
\hline Univariate analysis & & & 0.001 \\
Diabetes & 4.64 & $2.18-9.90$ & 0.001 \\
CK-MB [ng/mL] & 1.01 & $1.0-1.01$ & 0.001 \\
LA function & & & 0.01 \\
LA max [mL/m ${ }^{2}$ ) & 1.18 & $1.11-1.26$ & 0.003 \\
LA total ejection fraction [\%] & 0.93 & $0.88-0.98$ & 0.01 \\
LA active emptying fraction [\%] & 0.92 & $0.87-0.97$ & 0.001 \\
LA reservoir function [\%] & 0.98 & $0.97-0.99$ & 0.006 \\
PALS [\%] & 0.71 & $0.64-0.79$ & 0.001 \\
LV function & & & 0.004 \\
WMSI & 10.70 & $1.95-58.82$ & 0.005 \\
GLS [\%] & 1.81 & $1.50-2.18$ & 0.003 \\
GCS [\%] & 1.21 & $1.06-1.37$ & 0.001 \\
Multivariate analysis & & & $0.63-14.87$ \\
Diabetes & 4.93 & $0.68-0.87$ & $1.11-1.67$ \\
PALS [\%] & 0.77 & 1.36 & \\
GLS [\%] & & & \\
\hline
\end{tabular}

$\mathrm{Cl}$ - confidence interval; CK — creatine kinase; GCS - global circumferential strain; GLS — global longitudinal strain; LA — left atrium; LV - left ventricular; OR — odds ratio; PALS - peak atrial longitudinal strain; WMSI — wall motion score index

GLS > $-12.3 \%\left(\log -\operatorname{rank} \chi^{2}=37.3, \mathrm{p}=0.001\right)$ and PALS $<23.8 \%\left(\log -\operatorname{rank} \chi^{2}=47.0, \mathrm{p}=0.001\right)$, and had composite event rates of $3 \%$ and $4 \%$, respectively.

\section{Discussion}

The major results of this study showed the prognostic value of LV GLS and PALS measured 

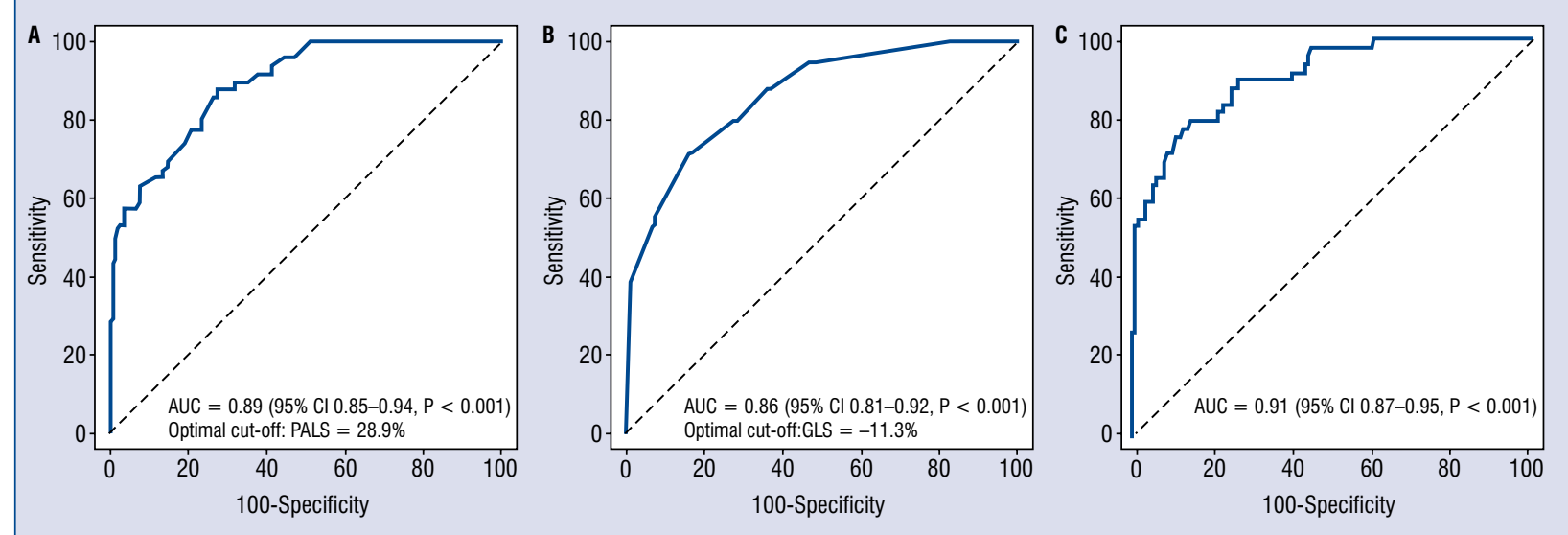

Figure 2. Receiver operating-characteristic curve for prediction of left ventricular remodeling 6 months after acute myocardial infarction using the independent variable peak atrial longitudinal strain (PALS) (A), left ventricular global longitudinal strain (LV GLS) (B) and PALS combined with GLS (C); AUC — area under curve.

Table 3. Baseline characteristics of patients, event and event-free.

\begin{tabular}{|c|c|c|c|}
\hline Parameter & Event-free & Event & $\mathbf{P}$ \\
\hline \multicolumn{4}{|l|}{ Clinical parameters } \\
\hline Male & $72 \%$ & $87 \%$ & 0.21 \\
\hline Age [years] & $57.4 \pm 10.5$ & $57.7 \pm 11.4$ & 0.90 \\
\hline $\mathrm{BMI}\left[\mathrm{kg} / \mathrm{m}^{2}\right]$ & $24.4 \pm 3.5$ & $25.3 \pm 5.0$ & 0.25 \\
\hline Hypertension & $74(42.0 \%)$ & $9(39.1 \%)$ & 0.83 \\
\hline Hyperlipidemia & $59(35.5 \%)$ & $12(52.2 \%)$ & 0.10 \\
\hline Smoking & $116(65.9 \%)$ & $18(78.2 \%)$ & 0.34 \\
\hline Systolic BP [mmHg] & $110.4 \pm 16.3$ & $115.6 \pm 21.3$ & 0.17 \\
\hline Diastolic BP [mmHg] & $72.0 \pm 13.7$ & $73.1 \pm 14.2$ & 0.71 \\
\hline Heart rate $[\mathrm{bpm}]$ & $74.6 \pm 14.1$ & $73.0 \pm 11.3$ & 0.61 \\
\hline QRS width [ms] & $97.4 \pm 15.9$ & $110.9 \pm 25.2$ & 0.001 \\
\hline S-TO-B [min] & $334.5 \pm 176.4$ & $397.2 \pm 162.3$ & 0.11 \\
\hline D-TO-B [min] & $48.9 \pm 19.5$ & $56.1 \pm 19.0$ & 0.10 \\
\hline eGFR $\left[\mathrm{mL} / \mathrm{min} / 1.73 \mathrm{~m}^{2}\right]$ & $93.5 \pm 26.2$ & $96.2 \pm 38.3$ & 0.66 \\
\hline Creatinine $[\mu \mathrm{mol} / \mathrm{L}]$ & $71.4 \pm 24.7$ & $66.6 \pm 11.9$ & 0.36 \\
\hline Grace (scores) & $96.6 \pm 25.4$ & $95.2 \pm 28.9$ & 0.79 \\
\hline Crusade (scores) & $21.8 \pm 12.9$ & $22.7 \pm 12.4$ & 0.78 \\
\hline Killip class $\geq$ II & $14(8.0 \%)$ & $6(26.1 \%)$ & 0.007 \\
\hline Anterior wall MI & $83(47.2 \%)$ & $20(87.0 \%)$ & 0.001 \\
\hline CK-MB [ng/mL] & $347.4 \pm 146.5$ & $437.2 \pm 98.5$ & 0.005 \\
\hline CK-MB peak time after onset [h] & $16.2 \pm 5.3$ & $18.4 \pm 5.1$ & 0.06 \\
\hline ST max before $\mathrm{PCl}[\mathrm{mm}]$ & $4.0 \pm 2.1$ & $3.7 \pm 1.9$ & 0.59 \\
\hline Multivessel coronary disease & $54(30 \%)$ & $8(35 \%)$ & 0.81 \\
\hline \multicolumn{4}{|l|}{ LA function } \\
\hline $\mathrm{LA} \max \left[\mathrm{mL} / \mathrm{m}^{2}\right]$ & $27.7 \pm 5.8$ & $33.1 \pm 7.5$ & 0.001 \\
\hline LA total ejection fraction [\%] & $54.7 \pm 5.9$ & $50.7 \pm 5.3$ & 0.002 \\
\hline LA passive emptying fraction [\%] & $28.6 \pm 7.6$ & $25.6 \pm 7.9$ & 0.07 \\
\hline LA active emptying fraction [\%] & $36.4 \pm 6.5$ & $33.6 \pm 4.4$ & 0.04 \\
\hline
\end{tabular}


Table 3 (cont.). Baseline characteristics of patients, event and event-free.

\begin{tabular}{lccc}
\hline Parameter & Event-free & Event & P \\
\hline LA reservoir function [\%] & $124.8 \pm 30.6$ & $105.8 \pm 22.4$ & 0.003 \\
Moderate or severe MR & $8(5 \%)$ & $2(9 \%)$ & 0.07 \\
PALS [\%] & $31.1 \pm 5.9$ & $22.7 \pm 5.7$ & 0.001 \\
Initial LV function & & & \\
LVESV [mL] & $88.3 \pm 21.7$ & $113.5 \pm 34.0$ & 0.001 \\
LVEDV [mL] & $42.9 \pm 13.4$ & $61.5 \pm 17.6$ & 0.001 \\
LVEF [\%] & $51.9 \pm 5.1$ & $46.6 \pm 4.0$ & 0.002 \\
GLS [\%] & $-14.1 \pm 3.1$ & $-10.2 \pm 1.9$ & 0.001 \\
GCS [\%] & $-14.2 \pm 3.3$ & $-12.6 \pm 3.5$ & 0.03 \\
\hline
\end{tabular}

Data are expressed as mean \pm standard deviation or number (\%). BMI — body mass index; BP — blood pressure; CK — creatine kinase; D-TO-B - door-to-balloon time; eGFR - estimated glomerular filtration rate; GCS - global circumferential strain; GLS - global longitudinal strain; LA - left atrium; LV — left ventricular; LVEDV — left ventricular end-diastolic volume; LVEF — left ventricular ejection fraction; LVESV - left ventricular end-systolic volume; MI - myocardial infarction; MR - mitral regurgitation; PALS — peak atrial longitudinal strain; $\mathrm{PCl}$ - percutaneous coronary intervention; ST max - maximum ST-segment elevation from a single lead; S-TO-B - symptom-to-balloon time

Table 4. Factors predicting adverse events according to Cox proportional hazards regression model using univariable and multivariate analysis.

\begin{tabular}{lccc}
\hline Parameters & HR & $95 \%$ Cl & P \\
\hline Univariate analysis & & & 0.001 \\
Diabetes & 4.96 & $2.18-11.2$ & 0.007 \\
CK-MB [ng/mL] & 1.01 & $1.00-1.01$ & 0.001 \\
LA max [mL/m²] & 1.14 & $1.08-1.20$ & 0.01 \\
LA total ejection fraction [\%] & 0.90 & $0.84-0.96$ & 0.04 \\
LA active emptying fraction [\%] & 0.93 & $0.87-0.99$ & 0.01 \\
LA reservoir function [\%] & 0.97 & $0.96-0.99$ & 0.001 \\
PALS [\%] & 0.82 & $0.76-0.88$ & 0.001 \\
LVEF [\%] & 0.82 & $0.76-0.89$ & 0.001 \\
GLS [\%] & 1.55 & $1.31-1.83$ & 0.02 \\
GCS (\%] & 1.08 & $1.01-1.16$ & 0.04 \\
Multivariate analysis & & & 0.03 \\
PALS [\%] & 0.88 & $0.78-0.99$ & 0.008 \\
GLS [\%] & 1.30 & $1.01-1.66$ & $1.50-14.19$ \\
Diabetes & 4.61 & & \\
\hline
\end{tabular}

$\mathrm{Cl}$ — confidence interval; CK — creatine kinase; GCS - global circumferential strain; GLS — global longitudinal strain; HR - hazard ratio; LA - left atrium; LV — left ventricular ejection fraction; PALS — peak atrial longitudinal strain

by 2D-STE in patients with STEMI after pPCI, as follows: (1) reductions in PALS and LV GLS are both strongly correlated to LV remodeling and the composite event; (2) however, PALS does not add significant incremental prognostic value to LV GLS.

Acute myocardial infarction is characterized by regional myocardial damage that results in systolic and diastolic dysfunction with a risk of adverse LV remodeling. For several decades, previous researchers have focused on the pathophysiology and prognosis of LV systolic dysfunction after AMI and have shown that LV remodeling mostly occurs in cases of transmural infarction and if at least $20 \%$ of LV mass is destroyed [3]. Although LVEF and WMSI have traditionally been used to evaluate the degree of myocardium injury and even WMSI is considered an independent predictor of LV remodeling [20, 21], either of them has 


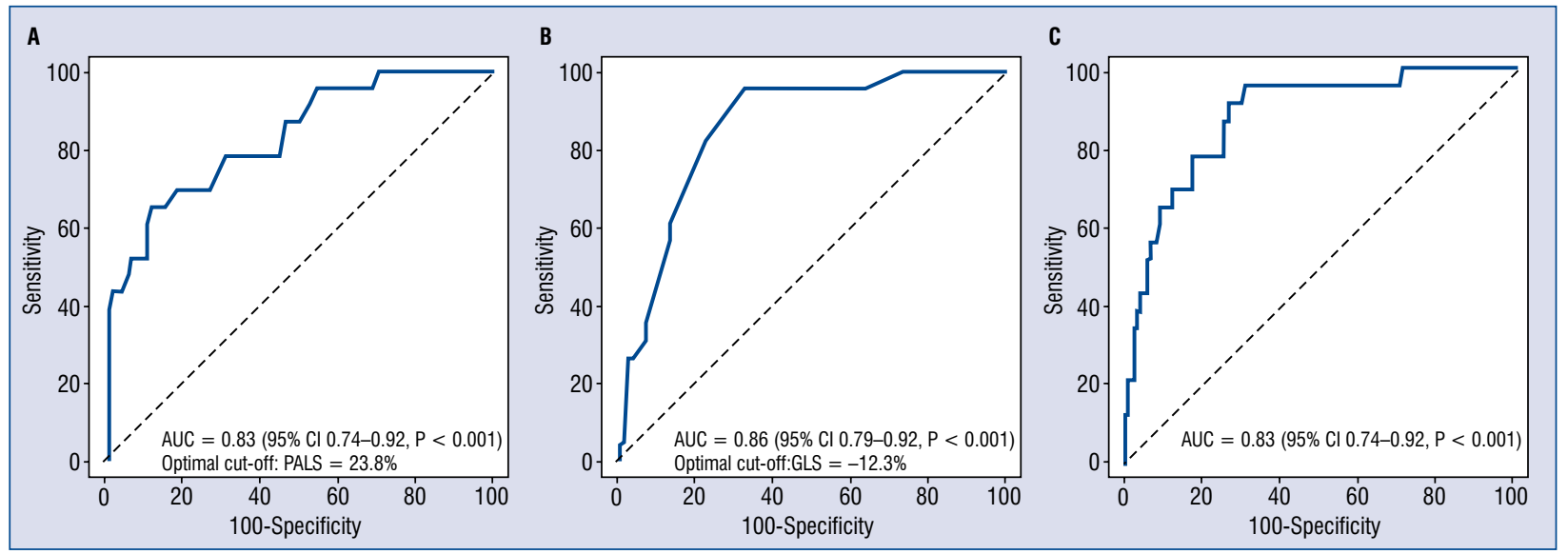

Figure 3. Receiver operating-characteristic curve for prediction of clinical adverse events using the peak atrial longitudinal strain (PALS) (A), left ventricular global longitudinal strain (LV GLS) (B) and PALS combined with GLS (C).

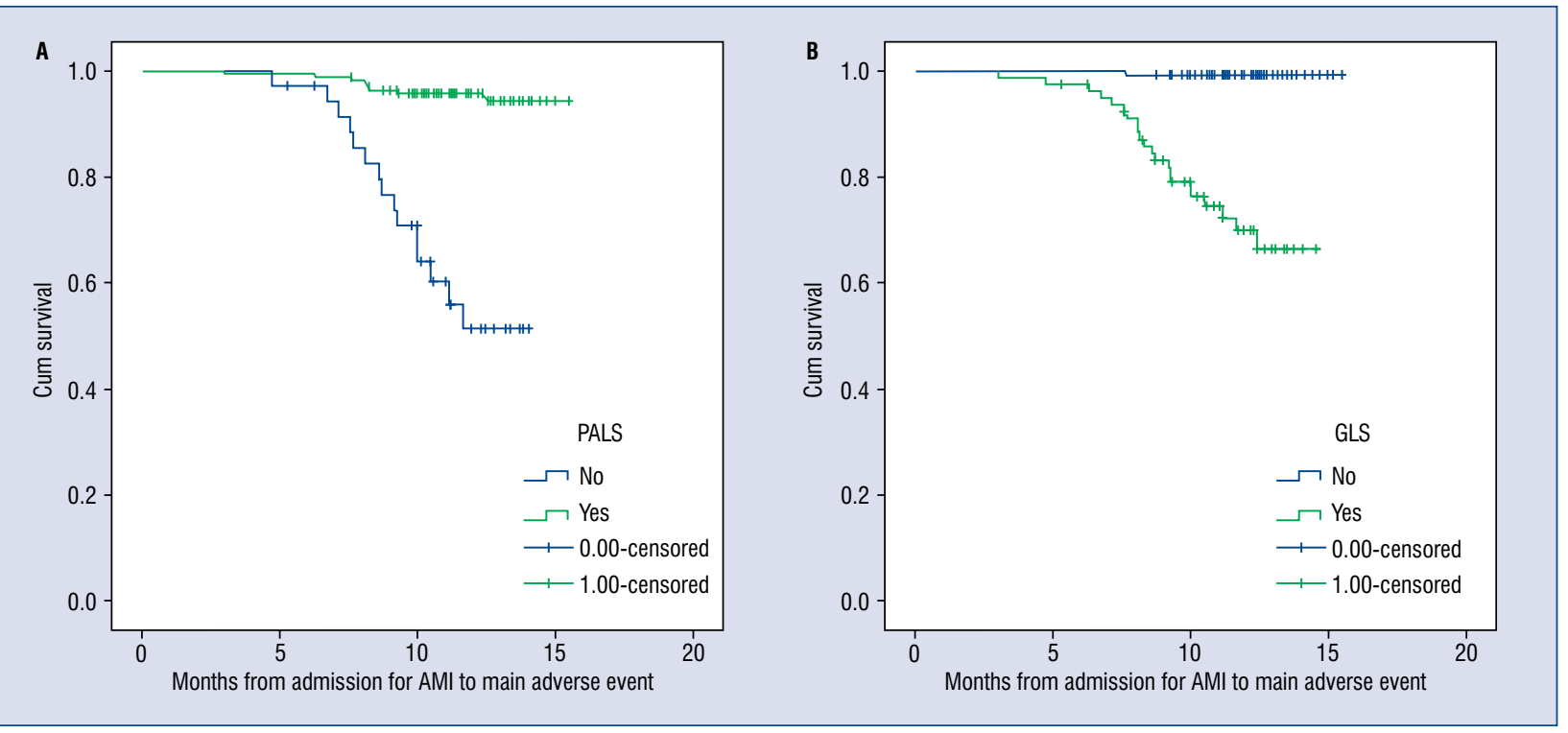

Figure 4. Survival analysis according to peak atrial longitudinal strain (PALS) and global longitudinal strain (GLS) values Kaplan-Meier survival curves for patients according to PALS (the optimal cutoff 23.8\%) (A) and left ventricular GLS (the optimal cutoff $-12.3 \%$ ) (B); AMI - acute myocardial infarction.

limitations for risk stratification after AMI [22]. 2D-STE, as a semiautomatic method, is not only applied to estimate the motion of the myocyte but also can distinguish the passive and active motility of LV segments, suggesting it is a more sensitive measurement of LV function [23]. The present results showed that LV GLS not LVEF and WMSI is an independent predictor of $\mathrm{LV}$ remodeling, and the AUC was 0.86 , and the best cutoff value was $-11.3 \%$, which is similar to the $-12.46 \%$ reported by Lacalzada et al. [24]. This may be because strain can better distinguish between passive and active motion of each segment of LV, and hence GLS appears to be more useful than LVEF and WMSI in predicting LV remodeling. Hung et al. [10] found that not only GLS but also GCS and circumferential strain rate are independent predictors of LV remodeling at 20 months after adjusting for clinical variables. It seems that circumferential function plays an essential role in maintaining $\mathrm{LV}$ structure, so circumferential dysfunction would lead to LV dilatation. In the current study, GCS 
was not an independent predictor by multivariate analysis. The reason for the contradictory data in predicting LV remodeling by GCS may be the different follow-up periods after AMI.

Park et al. [7] demonstrated that not only GLS showed good predictive value for LV remodeling in patients with anterior wall AMI but also predicted death or heart failure as composite events, indicating that GLS was also a good predictor of adverse clinical events. A previous study confirmed that LV strain and strain rate were superior to LVEF and WMSI in risk stratification for long-term outcome, and a GLS value $>-15.1 \%$ was an independent predictor of all-cause mortality [25]. However, the VALIANT Echo study, in a sample of 603 patients with LV dysfunction, heart failure, or both 5 days after myocardial infarction, showed that both longitudinal and circumferential strain and strain rate are the independent prognostic indicators in patients with high-risk myocardial infarction [10]. In the present study, it was shown that GLS is an independent predictor and the optimal GLS cutoffs for predicting composite events is $>-12.3 \%$, with a sensitivity and specificity of $95.7 \%$ and $67.0 \%$.

Currently, LA function is assessed by LA volume, mechanical function and strain. Previous observation reported that LA volume is significantly related to cardiovascular disease and is independently correlated to death or heart failure [26]. LA mechanical function consists of the reservoir function, conduit and contractile function. LA reservoir function, which reflects LA relaxation, is particularly important during acute ischemia [27]. However, assessing changes in LA volume during different periods of the cardiac cycle is highly time-consuming; in addition, applying a simple geometric model to an asymmetric chamber may affect the estimation of LA volume [28]. Recently, by directly evaluating LA myocardial deformation to assess LA reservoir function post-AMI, clinically relevant information can be provided. PALS, which is evaluated by speckle-tracking derived strain, shows the direct evaluation of the atrial myocardium and may better reflect the properties of LA [29, 30]. Antoni et al. [31] confirmed the value of PALS to predict adverse events in patients after AMI treated with PCI, since only 48 of 320 patients (15\%) reached the composite endpoint. This event rate was higher than the rate herein, where 23 of 199 patients (11.6\%) experienced these events, perhaps due to a significantly shorter follow-up time. However, Ersboll et al. [16] found that the magnitude of PALS during the reservoir phase depends on the GLS and LA size, and measurement of PALS has no independent prognostic value. In patients with post-AMI, LA relaxation may be damaged by myocyte loss and LV filling pressure may also increase, both of which may be present, possibly limiting atrial expansion independently of LV longitudinal contraction damage, consequently increasing the risk of $\mathrm{LV}$ remodeling and adverse events [31,32]. In the present study, PALS, like LV GLS, was found to be another independent predictor of $\mathrm{LV}$ remodeling; and a higher PALS value $<23.8 \%$, with a sensitivity and specificity of $88.1 \%$ and $65.2 \%$, was shown to be an independent predictor of a composite event.

In the current study, the independent prognostic value of PALS and LV GLS in patients with STEMI after pPCI was observed. Additionally, PALS did not add significant incremental value beyond $\mathrm{LV}$ GLS in the prediction of LV remodeling (AUC: 0.05 , $\mathrm{p}=0.24$ ) and clinical events (even a decrease in AUC: $0.03, p=0.69$ ). The highly predictive values of GLS and PALS are further underscored.

\section{Limitations of the study}

A number of limitations of this study should be acknowledged. First, this is a single-center experience. In addition, the enrolled population was limited to patients with their first STEMI treated with pPCI, with low-risk AMI, and patients who died before completing their 6-month echocardiogram were excluded. Therefore, selection bias and potential selection bias should be taken into account when interpreting the findings. Finally, although the longitudinal, circumferential and radial strain of LV was analyzed, the impairment of right ventricular function was not assessed, which needs further study.

\section{Conclusions}

In conclusion, in patients with STEMI in any location treated with pPCI, both LV GLS and PALS are both more sensitive to myocardial damage and provide independent prognostic value for adverse LV remodeling and clinical events. However, the ability of the combination of PALS and GLS to predict LV remodeling and clinical outcomes may not be superior to that of a single indicator.

\section{Conflict of interest: None declared}

\section{References}

1. Gaudron P, Eilles C, Kugler I, et al. Progressive left ventricular dysfunction and remodeling after myocardial infarction. Potential 
mechanisms and early predictors. Circulation. 1993; 87(3): 755-763, doi: 10.1161/01.cir.87.3.755, indexed in Pubmed: 8443896.

2. Bolognese L, Neskovic AN, Parodi G, et al. Left ventricular remodeling after primary coronary angioplasty: patterns of left ventricular dilation and long-term prognostic implications. Circulation. 2002; 106(18): 2351-2357, doi: 10.1161/01. cir.0000036014.90197.fa, indexed in Pubmed: 12403666.

3. Pfeffer MA, Braunwald E. Ventricular remodeling after myocardial infarction. Experimental observations and clinical implications. Circulation. 1990; 81(4): 1161-1172, doi: 10.1161/01. cir.81.4.1161, indexed in Pubmed: 2138525.

4. St John Sutton M, Lee D, Rouleau JL, et al. Left ventricular remodeling and ventricular arrhythmias after myocardial infarction. Circulation. 2003; 107(20): 2577-2582, doi: 10.1161/01. CIR.0000070420.51787.A8, indexed in Pubmed: 12732606.

5. Lang RM, Bierig M, Devereux RB, et al. Recommendations for chamber quantification: a report from the American Society of Echocardiography's Guidelines and Standards Committee and the Chamber Quantification Writing Group, developed in conjunction with the European Association of Echocardiography, a branch of the European Society of Cardiology. J Am Soc Echocardiogr. 2005; 18(12): 1440-1463, doi: 10.1016/j.echo.2005.10.005, indexed in Pubmed: 16376782.

6. Mor-Avi V, Lang RM, Badano LP, et al. Current and Evolving Echocardiographic Techniques for the Quantitative Evaluation of Cardiac Mechanics: ASE/EAE Consensus Statement on Methodology and Indications Endorsed by the Japanese Society of Echocardiography. European Journal of Echocardiography. 2011; 12(3): 167-205, doi: 10.1093/ejechocard/jer021.

7. Park YH, Kang SJ, Song JK, et al. Prognostic value of longitudinal strain after primary reperfusion therapy in patients with anterior-wall acute myocardial infarction. J Am Soc Echocardiogr. 2008; 21(3): 262-267, doi: 10.1016/j.echo.2007.08.026, indexed in Pubmed: 17904803.

8. Cimino S, Canali E, Petronilli V, et al. Global and regional longitudinal strain assessed by two-dimensional speckle tracking echocardiography identifies early myocardial dysfunction and transmural extent of myocardial scar in patients with acute ST elevation myocardial infarction and relatively preserved LV function. Eur Heart J Cardiovasc Imaging. 2013; 14(8): 805-811, doi: 10.1093/ehjci/jes295, indexed in Pubmed: 23258316.

9. Hoogslag GE, Abou R, Joyce E, et al. Comparison of changes in global longitudinal peak systolic strain after ST-segment elevation myocardial infarction in patients with versus without diabetes mellitus. Am J Cardiol. 2015; 116(9): 1334-1339, doi: 10.1016/j.amjcard.2015.07.061, indexed in Pubmed: 26341185.

10. Hung CL, Verma A, Uno H, et al. VALIANT investigators. Longitudinal and circumferential strain rate, left ventricular remodeling, and prognosis after myocardial infarction. J Am Coll Cardiol. 2010; 56(22): 1812-1822, doi: 10.1016/j.jacc.2010.06.044, indexed in Pubmed: 21087709.

11. Mollema SA, Nucifora G, Bax JJ. Prognostic value of echocardiography after acute myocardial infarction. Heart. 2009; 95(21): 1732-1745, doi: 10.1136/hrt.2008.161836, indexed in Pubmed: 19276097.

12. Hoit BD. Left atrial size and function: role in prognosis. J Am Coll Cardiol. 2014; 63(6): 493-505, doi: 10.1016/j.jacc.2013.10.055, indexed in Pubmed: 24291276.

13. Todaro MC, Choudhuri I, Belohlavek M, et al. New echocardiographic techniques for evaluation of left atrial mechanics. Eur
Heart J Cardiovasc Imaging. 2012; 13(12): 973-984, doi: 10.1093/ ehjci/jes174, indexed in Pubmed: 22909795.

14. Cameli M, Lisi M, Focardi M, et al. Left atrial deformation analysis by speckle tracking echocardiography for prediction of cardiovascular outcomes. Am J Cardiol. 2012; 110(2): 264-269, doi: 10.1016/j.amjcard.2012.03.022, indexed in Pubmed: 22497676.

15. Freed BH, Daruwalla V, Cheng JY, et al. Prognostic Utility and Clinical Significance of Cardiac Mechanics in Heart Failure With Preserved Ejection Fraction: Importance of Left Atrial Strain. Circ Cardiovasc Imaging. 2016; 9(3), doi: 10.1161/CIRCIMAGING.115.003754, indexed in Pubmed: 26941415.

16. Ersbøll M, Andersen MJ, Valeur N, et al. The prognostic value of left atrial peak reservoir strain in acute myocardial infarction is dependent on left ventricular longitudinal function and left atrial size. Circ Cardiovasc Imaging. 2013; 6(1): 26-33, doi: 10.1161/ /CIRCIMAGING.112.978296, indexed in Pubmed: 23192848.

17. O'Gara PT, Kushner FG, Ascheim DD, et al. 2013 ACCF/AHA guideline for the management of ST-elevation myocardial infarction: executive summary: a report of the American College of Cardiology Foundation/American Heart Association Task Force on Practice Guidelines: developed in collaboration with the American College of Emergency Physicians and Society for Cardiovascular Angiography and Interventions. Catheter Cardiovasc Interv. 2013; 82(1): E1-27, doi: 10.1002/ccd.24776, indexed in Pubmed: 23299937.

18. Leung DY, Boyd A, Ng AA, et al. Echocardiographic evaluation of left atrial size and function: current understanding, pathophysiologic correlates, and prognostic implications. Am Heart J. 2008; 156(6): 1056-1064, doi: 10.1016/j.ahj.2008.07.021, indexed in Pubmed: 19032999.

19. Alpert JS, Thygesen K, Antman E, et al. Myocardial infarction redefined--a consensus document of The Joint European Society of Cardiology/American College of Cardiology Committee for the redefinition of myocardial infarction. Eur Heart J. 2000; 21(18): 1502-1513, doi: 10.1053/euhj.2000.2305, indexed in Pubmed: 10973764.

20. Bochenek T, Wita K, Tabor Z, et al. Value of speckle-tracking echocardiography for prediction of left ventricular remodeling in patients with ST-elevation myocardial infarction treated by primary percutaneous intervention. J Am Soc Echocardiogr. 2011; 24(12): 1342-1348, doi: 10.1016/j.echo.2011.09.003, indexed in Pubmed: 22000785.

21. O'Gara PT, Kushner FG, Ascheim DD, et al. 2013 ACCF/AHA guideline for the management of ST-elevation myocardial infarction: executive summary: a report of the American College of Cardiology Foundation/American Heart Association Task Force on Practice Guidelines. J Am Coll Cardiol. 2013; 61(4): 485-510, doi: 10.1016/j.jacc.2012.11.018, indexed in Pubmed: 23256913.

22. Chen X, Nakatani S. Transmural myocardial strain gradient: a new and robust quantitative index of left ventricular wall motion based on myocardial strain imaging. Echocardiography. 2011; 28(2): 181-187, doi: 10.1111/j.1540-8175.2010.01287.x, indexed in Pubmed: 21276074.

23. Edvardsen T, Gerber BL, Garot J, et al. Quantitative assessment of intrinsic regional myocardial deformation by Doppler strain rate echocardiography in humans: validation against threedimensional tagged magnetic resonance imaging. Circulation. 2002; 106(1): 50-56, doi: 10.1161/01.cir.0000019907.77526.75, indexed in Pubmed: 12093769.

24. Lacalzada J, de la Rosa A, Izquierdo MM, et al. Left ventricular global longitudinal systolic strain predicts adverse remodeling 
and subsequent cardiac events in patients with acute myocardial infarction treated with primary percutaneous coronary intervention. Int J Cardiovasc Imaging. 2015; 31(3): 575-584, doi: 10.1007/s10554-015-0593-2, indexed in Pubmed: 25596940.

25. Antoni ML, Mollema SA, Delgado V, et al. Prognostic importance of strain and strain rate after acute myocardial infarction. Eur Heart J. 2010; 31(13): 1640-1647, doi: 10.1093/eurheartj/ehq105, indexed in Pubmed: 20423918.

26. Pritchett AM, Jacobsen SJ, Mahoney DW, et al. Left atrial volume as an index of left atrial size: a population-based study. J Am Coll Cardiol. 2003; 41(6): 1036-1043, doi: 10.1016/s07351097(02)02981-9, indexed in Pubmed: 12651054.

27. Stefanadis C, Dernellis J, Toutouzas P. A clinical appraisal of left atrial function. Eur Heart J. 2001; 22(1): 22-36, doi: 10.1053/ /euhj.1999.2581, indexed in Pubmed: 11133207.

28. Yagmur J, Cansel M, Kurtoglu E, et al. Assessment of left atrial volume and function by real time three-dimensional echocardiography in obese patients. Echocardiography. 2017; 34(2): 210-216, doi: 10.1111/echo.13417, indexed in Pubmed: 27933639.

29. Motoki H, Dahiya A, Bhargava M, et al. Assessment of left atrial mechanics in patients with atrial fibrillation: comparison between two-dimensional speckle-based strain and velocity vector imaging. J Am Soc Echocardiogr. 2012; 25(4): 428-435, doi: 10.1016/j. echo.2011.12.020, indexed in Pubmed: 22265458.

30. Vianna-Pinton R, Moreno CA, Baxter CM, et al. Two-dimensional speckle-tracking echocardiography of the left atrium: feasibility and regional contraction and relaxation differences in normal subjects. J Am Soc Echocardiogr. 2009; 22(3): 299 -305, doi: 10.1016/j.echo.2008.12.017, indexed in Pubmed: 19258177.

31. Antoni ML, ten Brinke EA, Atary JZ, et al. Left atrial strain is related to adverse events in patients after acute myocardial infarction treated with primary percutaneous coronary intervention. Heart. 2011; 97(16): 1332-1337, doi: 10.1136/hrt.2011.227678, indexed in Pubmed: 21613636.

32. Temporelli PL, Giannuzzi P, Nicolosi GL, et al. GISSI-3 Echo Substudy Investigators. Doppler-derived mitral deceleration time as a strong prognostic marker of left ventricular remodeling and survival after acute myocardial infarction: results of the GISSI-3 echo substudy. J Am Coll Cardiol. 2004; 43(9): 1646-1653, doi: 10.1016/j.jacc.2003.12.036, indexed in Pubmed: 15120826. 